\title{
Protective effects of astaxanthin against ischemia/ reperfusion induced renal injury in mice
}

\author{
Xuefeng Qiu ${ }^{1,2,3 \dagger}$, Kai Fu ${ }^{1,2+}$, Xiaozhi Zhao ${ }^{1,2+}$, Yanting Zhang ${ }^{3}$, Yimin Yuan ${ }^{1}$, Shiwei Zhang ${ }^{1}$, Xiaoping Gu ${ }^{*}$ \\ and Hongqian Guo ${ }^{1 *}$
}

\begin{abstract}
Astaxanthin (ATX) is a powerful antioxidant that occurs naturally in a wide variety of living organisms. Previous studies have shown that ATX has effects of eliminating oxygen free radicals and can protect organs from ischemia/ reperfusion (IR) induced injury. The present study was designed to further investigate the protective effects of ATX on oxidative stress induced toxicity in tubular epithelial cells and on IR induced renal injury in mice. ATX, at a concentration of $250 \mathrm{nM}$, attenuated $100 \mathrm{\mu M} \mathrm{H}_{2} \mathrm{O}_{2}$-inudced viability decrease of tubular epithelial cells. In vivo, ATX preserved renal function $12 \mathrm{~h}$ or $24 \mathrm{~h}$ post IR. Pretreatment of ATX via oral gavage for 14 consecutive days prior to IR dramatically prevented IR induced histological damage $24 \mathrm{~h}$ post IR. Histological results showed that the pathohistological score, number of apoptotic cells, and the expression of a-smooth muscle actin were significantly decreased by pretreatment of ATX. In addition, oxidative stress and inflammation in kidney samples were significantly reduced by ATX $24 \mathrm{~h}$ post IR. Taken together, the current study suggests that pretreatment of ATX is effective in preserving renal function and histology via antioxidant activity.
\end{abstract}

Keywords: Renal transplantation, Kidney, Ischemia/reperfusion, Oxidative stress, Astaxanthin, Antioxidant

\section{Introduction}

Kidney transplantation presents one of the gold standard therapies for end-stage renal diseases. Given the storage of organs from decreased donors available for transplantation, transplants using so-called "marginal" organs have increased despite their reduced viability and increased immunogenicity compared with living donor renal allografts [1,2]. Ischemia/reperfusion (IR), an inevitable consequence of kidney transplantation, is one of the major contributors to the development of primary injury, and delayed graft function in kidney transplantation, leading to worse long-term function [3,4].

Although the mechanisms by which organ damage occurs in IR-induced renal injury are incompletely understood, it has been suggested that oxidative injury following IR most likely contributed to the pathogenesis of IR [5]. During the ischemia phase, ischemia-induced

\footnotetext{
* Correspondence: xiaopinggu1@gmail.com; dr.guohongqian@gmail.com ${ }^{\dagger}$ Equal contributors

${ }^{4}$ Department of Anesthesiology, Affiliated Drum Tower Hospital, School of Medicine, Nanjing University, Nanjing 210008, China

'Department of Urology, Affiliated Drum Tower Hospital, School of Medicine, Nanjing University, Nanjing 210008, China

Full list of author information is available at the end of the article
}

hypoxia could induce ATP depletion and hypoxanthine accumulation. After reintroduction of blood flow, reactive oxygen species (ROS) are generated [6], leading to tubular apoptosis/necrosis and local inflammation [7]. As a result of oxidative and inflammatory injury caused by IR, tubular epithelial cells might undergo profound functional alterations including losing tight junctions and epithelial-to-mesenchymal transition (EMT) $[8,9]$. Previous reports have demonstrated that deficiency of antioxidant could exacerbate IR injury and antioxidants such as superoxide dismutase, catalase, or vitamin E had beneficial effects for IR injury [10,11], supporting oxidative stress as a major contributor in the development of IR-induced injury.

Astaxanthin (ATX) is a carotenoid pigment naturally existing in seafood, such as salmon, shells of crabs and shrimps, as well as a wide variety of plants and algae [12]. The United States FDA approved ATX as a feed additive for the aquaculture industry in 1987 and approved its use as a dietary supplement in 1999 [13]. The molecular structure of ATX is similar to that of $\beta$ carotene (Additional file 1: Figure S1), which gives ATX antioxidant capacity [14]. ATX exhibits free radical 
scavenging activity and protect against lipid peroxidation and oxidative damage to cell membranes, cells, and tissues, suggesting ATX as a powerful biological antioxidant [15]. Unlike carotenoids, ATX has oxygenated groups on each ring structure (Additional file 1: Figure S1), making ATX highly polar and dramatically enhancing its membrane function to protect against degenerative conditions. This makes ATX a significantly greater antioxidant than $\beta$-carotene [16]. Previous studies on animal models have shown protective effects of ATX in IR-induced liver [17], brain $[18,19]$, or cardiovascular [20] injury by reducing oxidant-induced damage. However, the protective effect of ATX against IR-induced renal injury still remains unknown.

The present study was therefore designed to examine the therapeutic effect of ATX in IR-induced renal injury using an IR model in mice. We hypothesize that pretreatment of ATX could offer protective effects against IR-induced renal injury by reducing ROS, tubular apoptosis, inflammation, and EMT. To the best of our knowledge, this is the first study designed to explore the feasibility of using ATX, a powerful antioxidant, for IRinduced renal injury.

\section{Materials and methods}

\section{Cells culture and treatment}

Human tubular epithelial cells (HTECs) were obtained from Dr. Hao Chen (National Clinical Research Center of Kidney Diseases, Nanjing University, Nanjing, China) and cultured in Roswell Park Memorial Institute 1604 medium (RPMI 1640, Life Science, Shanghai, China) supplemented with $10 \%$ fetal bovine serum (FBS, Life Science). Culture incubator was set at $37^{\circ} \mathrm{C}$ with $5 \%$ $\mathrm{CO}_{2}$. In order to produce oxidative stress, $\mathrm{H}_{2} \mathrm{O}_{2}$ was freshly prepared prior to each experiment. Free type of ATX ( $\geq 97 \%$, Sigma-Aldrich, St. Louis, MO, USA) was dissolved in $0.5 \%$ DMSO. The cells were pretreated with ATX at concentration of $250 \mathrm{nM}$ for $24 \mathrm{~h}$ followed by exposure to $100 \mu \mathrm{M}$ of $\mathrm{H}_{2} \mathrm{O}_{2}$ in the presence of the same concentrations of ATX for another $2 \mathrm{~h}$.

\section{Cell viability assay}

Cell viability was evaluated by the Cell Counting Kit- 8 (CCK-8, Life Science) test. Five thousand cells were seeded into a 96-well culture plate and cultured in the serum-free growth medium. According to the instructions provided by the manufacturer, $100 \mu \mathrm{l}$ of CCK- 8 solution were added to each well and the cells were incubated for a further $2 \mathrm{~h}$. After indicated time points, absorbance was measured at $450 \mathrm{~nm}$ with a multidetection micro-plate reader (Versamax, Downingtown, PA, USA).

\section{Animals and treatment}

All experimental protocols conducted on animals were performed in accordance with the standards established by the Institution Animal Care and Use Committee at Nanjing University. Male ICR mice weighing 20-25 g were housed in stainless steel cages and given free access to food and water. Mice were randomly divided into four groups: The sham group received olive oil via oral gavage for 14 consecutive days [17] before the sham surgery $(\mathrm{n}=6)$; the sham + ATX group received $5 \mathrm{mg} / \mathrm{kg} /$ day ATX (3,3-dihydroxy beta, $\beta$-carotene-4,4-dione, SigmaAldrich) dissolved in olive oil before the sham surgery $(n=6)$; the IR group received olive oil before the induction of renal IR $(n=16)$; the IR + ATX group received ATX before the induction of renal IR $(n=16)$. Mice in IR groups were sacrificed $12 \mathrm{~h}$ or $24 \mathrm{~h}$ post IR while mice in sham groups were sacrificed $24 \mathrm{~h}$ post sham surgery. Blood samples were collected for measurement of renal function while kidneys were collected for molecular analysis.

\section{Mice model of renal IR injury}

Mice were anesthetized intraperitoneally with a mixture of ketamine $(100 \mathrm{mg} / \mathrm{kg})$ and midazolam $(5 \mathrm{mg} / \mathrm{kg})$. After anesthetization, mice were subjected to renal IR injury as previously described [21]. Briefly, after abdominal laparotomy, right kidney were exposed and removed. Left renal pedicle was exposed and clamped with vascular clamp for $45 \mathrm{~min}$ to induce ischemia. After clamp, vascular clamp was removed to induce reperfusion and the incision was closed in 2 layers. Sham-operated control animals underwent right kidney removal without occlusion of left renal artery.

\section{Measurement of renal function}

Serum were separated by centrifuging blood samples and stored at $-80^{\circ} \mathrm{C}$ until analysis of blood serum urea nitrogen (BUN) and urine creatinine $(\mathrm{Cr})$. The concentrations of BUN and $\mathrm{Cr}$ were assessed in duplicated with a commercially available assay kit (BioAssay System, Hayward, CA, USA) according to the instructions.

\section{Histological analysis}

Middle part of kidney were fixed in $4 \%$ formaldehyde, dehydrated, and paraffin embedded. Tissue sections $(5 \mu \mathrm{m})$ were stained with hematoxylin and eosin (HE). Kidney sections were examined in a blinded manner and scored to evaluate the degree of injury. The score reflected the grading of tubular necrosis, cast formation, tubular dilation, and loss of brush border in 10 randomly selected, non-overlapping fields (200X) as following: 0 , none; $1, \leq 10 \% ; 2,11$ to $25 \% ; 3,26$ to $45 \% ; 4,46$ to $75 \%$; and $5, \geq 76 \%[22,23]$.

\section{Immunofluorescent analysis}

After dewaxing and rehydration, the tissue sections were incubated with $3 \% \mathrm{BSA} / 0.3 \%$ Triton $\mathrm{X}-100$ for $30 \mathrm{~min}$ at 
room temperature. After draining this solution, the sections were incubated at room temperature with rabbit anti-myeloperoxidase (MPO, 1:100, Santa Cruz Biotechnologies, Santa Cruz, CA, USA), mouse anti- $\alpha$ smooth muscle actin ( $\alpha$-SMA) (1:100, Boster, Wuhan, China) overnight. Control tissue sections were similarly prepared except no primary antibody was added. After rinse with PBS, the sections were incubated with Alexa-594conjugated secondary antibody (Life Science). After rinses with PBS, the slides were incubated with 4',6-diamidino-2-phenylindole (DAPI, Life Science). The fluorescent was detected by confocal in darkness.

\section{Detection of apoptosis}

Quantitative determination of apoptosis in kidney sections was assessed by a terminal transferase-mediated dUTP nick-end labeling (TUNEL) assay using an In Situ Cell Death Detection Kit (Roche, Basel, Switzerland). After dewaxing and rehydration, the tissue sections were permeabilized with $0.1 \%$ Triton X-100 for $10 \mathrm{~min}$. Incubation with label solution was used to detect the apoptotic cells according to the instructions. Apoptotic score was achieved by counting the number of positive nuclei in 10 random fields.

\section{Measurement of superoxide dismutase (SOD) and malondialdehyde (MDA)}

Renal SOD and MDA levels were determined under the guidance of the kit instructions (Jiancheng Bioengineering Institute, Nanjing, China). Briefly, renal tissues were homogenized. After centrifugation, SOD was determined using the xanthine oxidase method while MDA was determined by the thiobarbituric acid (TBA) reaction. Each assay was repeated three times. Protein concentration was determined by the BCA Protein assay (Pierce, Rockford, IL) using bovine serum albumin (BSA) as a standard.

\section{Determination of myeloperoxidase (MPO) activity}

Commercial available kit (Jiancheng Bioengineering Institute) was used to detect MPO, an indicator of neutrophil infiltration, in the renal tissues according to the instruction. MPO activity was defined as the quantity of enzyme degrading $1 \mu \mathrm{mol}$ of peroxide per minute at $37^{\circ} \mathrm{C}$ and was expressed in unit per milligram protein.

\section{Enzyme-linked immunosorbent assay (ELISA)}

The supernatant from kidney homogenate was prepared for detecting the levels of TNF- $\alpha$, IL-1 $\beta$, and IL- 6 with a commercial available ELISA kit following the instructions of the manufacturer (Uscn Life Science Inc, Wuhan, China). The absorbance was read on a microplate reader and the concentrations were calculated according to the standard curve. Protein content in the sample was determined by Coomassie blue assay and the results were corrected per microgram of protein.

\section{Statistical analysis}

Data were analyzed using Prism 4 (GraphPad Software, San Diego, CA, USA) and expressed as mean \pm standard deviation. Multiple groups were compared using oneway analysis of variance followed by the Tukey-Kramer test for post-hoc comparisons. Statistical significance was set at $P<0.05$.

\section{Results}

ATX protects HETCs against $\mathrm{H}_{2} \mathrm{O}_{2}$-induced death

As shown in Figure 1, $\mathrm{H}_{2} \mathrm{O}_{2}$ decreased the cell viability in a dose dependent manner. The concentration of $100 \mu \mathrm{M}$ was further used for the determination of $\mathrm{H}_{2} \mathrm{O}_{2}$-inudced cell damage in the present experiments. ATX at different concentration $(250,500,1000,2000 \mathrm{nM})$ did not show toxic effect on the cells (Figure 1B). The concentration of $250 \mathrm{nM}$ was used for the further experiment. As shown in Figure 1C, ATX significantly increased the viability of HTECs exposed to $\mathrm{H}_{2} \mathrm{O}_{2}$.

\section{ATX preserves renal function and renal histology}

Two time points (12 h after and $24 \mathrm{~h}$ after IR procedure) were chosen to evaluate serial changes in serum levels of BUN and Cr. As shown in Figure 2A-B, both BUN and Cr levels were significantly higher in IR group than sham group and sham + ATX group. Pretreatment with ATX showed positive effect in preserving renal function, reflected by significantly reduced BUN and $\mathrm{Cr}$ levels in IR + ATX group. There was no significance between sham group and sham + ATX group.

To determine the effect of ATX on IR-induced renal injury, a histological scoring system base on the typical microscopic features of acute tubular damage including tubular necrosis and dilatation, cast formation, and loss of brush border was adopted (Additional file 1: Figure S2). At 24 hours after the IR procedure, the injury score was highest in IR group, significantly higher than sham group. The injury score in IR + ATX group was significantly reduced compared with IR group, suggesting the protective effect of ATX for IR-induced renal injury (Figure 2C-D).

\section{ATX alleviates oxidative stress}

Levels of SOD and MDA were used to evaluate oxidative stress in kidney. As shown in Figure 3, level of SOD was significantly higher while level of MDA was significantly lower in IR group compared with sham group, indicating oxidative stress in the kidney $24 \mathrm{~h}$ after IR procedure. Pretreatment of ATX reversed oxidative stress in IR + ATX group, reflected by significantly increased SOD level and decreased level of MDA compared with IR group. 

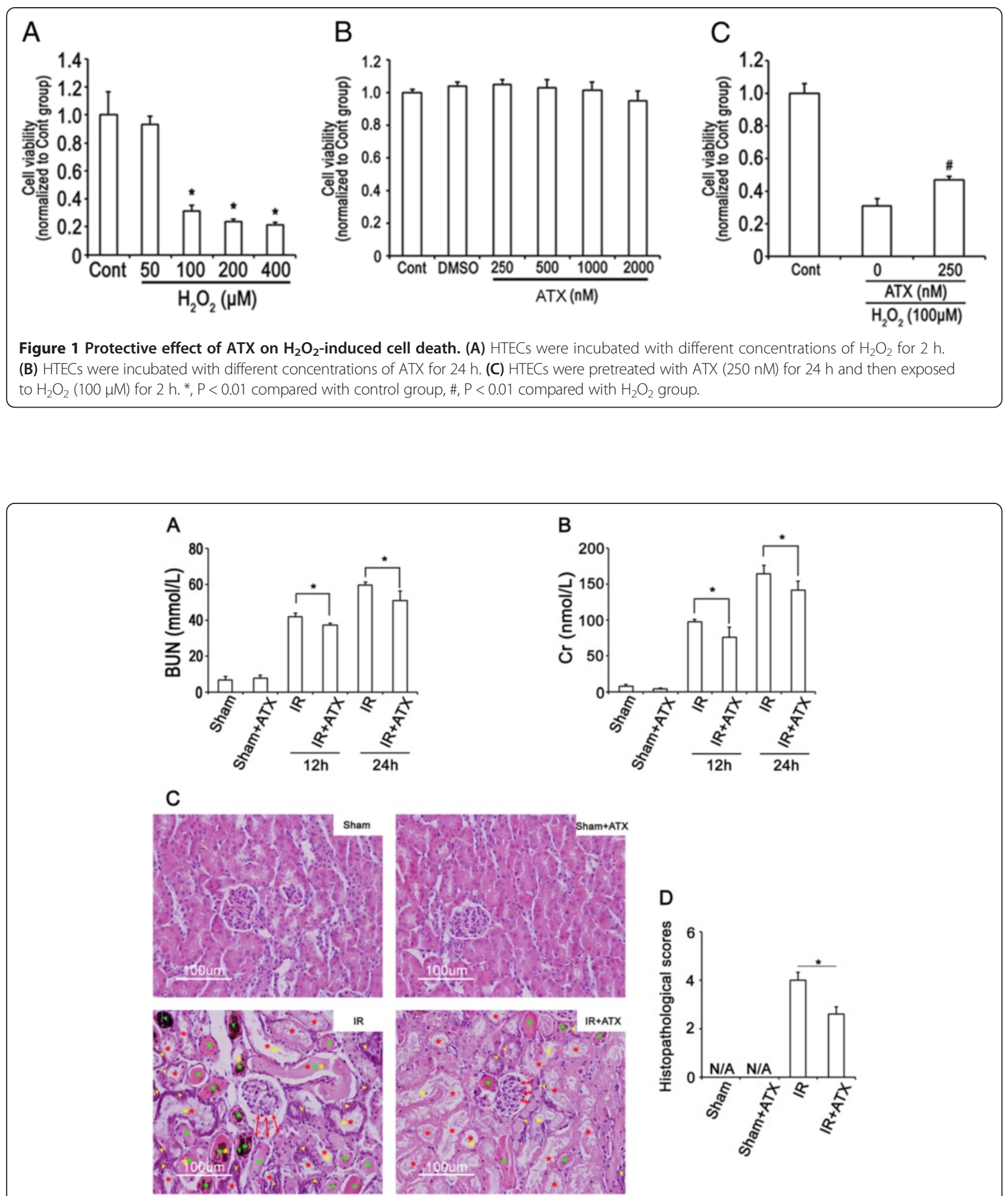

Figure 2 ATX preserves renal function and histology after IR. (A-B) Circulating levels of urea nitrogen (BUN) and creatinine (Cr) in different experimental groups ( $n=6$ in each group). (C) Representative images of HE staining of kidney sections in each experimental groups, showing significantly higher degree of tubular damage including tubular necrosis (yellow arrow), cast formation (green asterisk), dilatation of tubules (red asterisk), loss of brush border (yellow asterisk) in IR group. Please note that dilatation of Bowman's capsule (red arrow), and accumulation of red blood cells in tubules (green arrow) could also be observed in IR groups, suggesting the damage to glomerular. (D) Results of total histopathological scores reflecting tubular damage in each group. * $\mathrm{P}<0.05$ compared with IR group. 


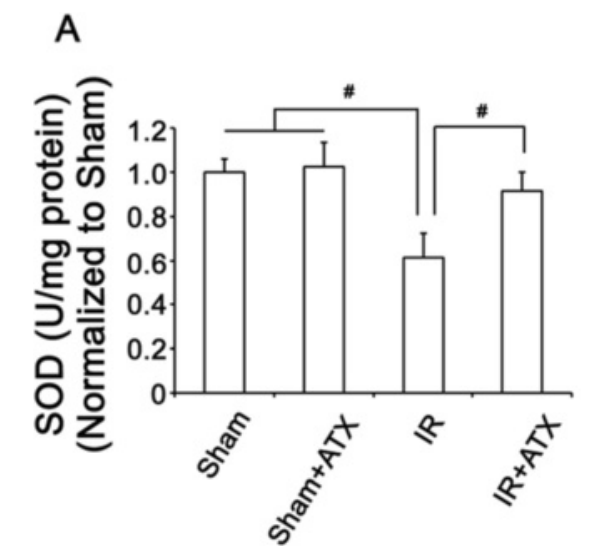

B

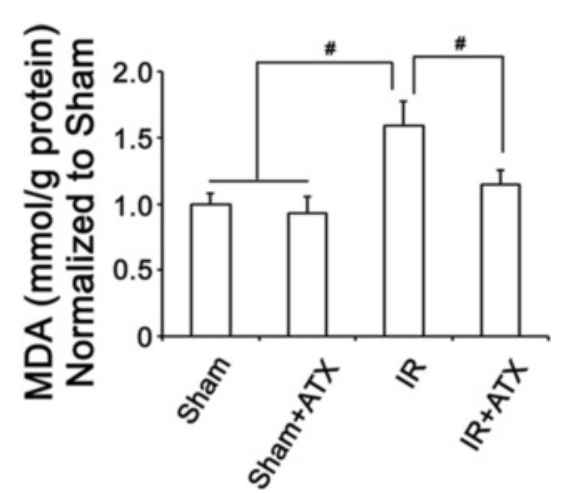

Figure 3 ATX alleviates oxidative stress after IR. Levels of SOD $(\mathbf{A})$ and MDA (B) in the renal tissue in each experimental group. ${ }^{*}, \mathbf{P}<0.05$ compared with IR group.

\section{Effects ATX on reducing apoptosis in kidney after IR procedure}

To investigate IR associated apoptotic cells, we measured TUNEL positive cells in kidney tissues. As shown in Figure 4, 24 hours after IR, no apoptotic cells were observed in the kidney from sham group and sham + ATX group. The number of apoptotic cells increased significantly in IR group compared with sham group. In contrast, tissues from IR + ATX group contain a significantly smaller number of TUNEL-positive apoptotic cells.

Effects of ATX on decreasing inflammation in kidney after IR MPO activity in kidney tissue and the protein levels of inflammatory cytokines including TNF- $\alpha$, IL-1 $\beta$, and IL6 were used to evaluate inflammation in kidneys after IR procedure. As shown in Figure 5A, the MPO activity in IR group was significantly higher than that in sham group $24 \mathrm{~h}$ after IR, indicating neutrophil infiltration after IR procedure. Compared with IR group, ATX pretreatment produced a significant decrease of MPO activity.

ELISA detection showed significantly increased expressions of TNF- $\alpha$, IL- $1 \beta$, and IL- 6 in IR group compared with that in sham group, suggesting inflammation in kidney $24 \mathrm{~h}$ after IR procedure. In the IR + ATX group, the levels of inflammatory cytokines decreased significantly compared with the IR group (Figure 5B).

\section{ATX reduces the expression of $a-S M A$ in tubular cells after IR}

$\alpha$-SMA positive myofibroblasts are the main actors in the pathogenesis of renal fibrosis. Because $\alpha$-SMA production is modulated by ROS in some cell lines, we investigated the expression of this myofibroblast marker in our mice model of IR injury. As shown in Figure 6, almost none $\alpha$-SMA expression could be detected within the interstitial space in the sham and sham + ATX group, while a significant increase in the expression of $\alpha$-SMA was observed within the tubular areas of renal tissues $24 \mathrm{~h}$ post IR procedure. In contrast, the expression of this marker was significantly decreased in the IR + ATX group.

\section{Discussion}

This study examined the effect of ATX pretreatment on IR-induced renal injury. Tissue distribution of ATX was studied previously in rodent after multiple-dosage regimens. The results revealed high accumulation of ATX in kidneys [24]. In the present study, ATX was dissolved in olive oil and administrated at a dosage of $5 \mathrm{mg} / \mathrm{kg} /$ day via oral gavage for 14 consecutive days before the induction of renal IR. The dosage of $5 \mathrm{mg} / \mathrm{kg} /$ day was selected based on our preliminary results that dosage of $10 \mathrm{mg} /$ $\mathrm{kg} /$ day or $20 \mathrm{mg} / \mathrm{kg} /$ day did not show significantly enhanced efficacy in ameliorating renal function (Additional file 1: Figure S2).

ATX pretreatment leaded to an impressive protection in IR induced renal injury reflected by significantly increased viability of HTECs (Figure 1) and lower total histopathological score (Figure 2). As stated previously, the total histopathological score was obtained by summation of all scores given to tubular necrosis, cast formation, tubular dilation, and loss of brush border [22]. The histopathological evaluation revealed that total injury score was significantly ameliorated in ATX pretreated kidneys underwent IR as compared to non pretreated control. BUN and $\mathrm{Cr}$, which are clinically used as markers to reflect renal function, were used in the present study to reflect renal function post IR. Although the functional differences between ATX pretreated group and non-treated control is significant (Figure 1), the decline of BUN or $\mathrm{Cr}$ in ATX pretreated groups was not consistent with that of histopathological score in ATX pretreated group. This might be because 

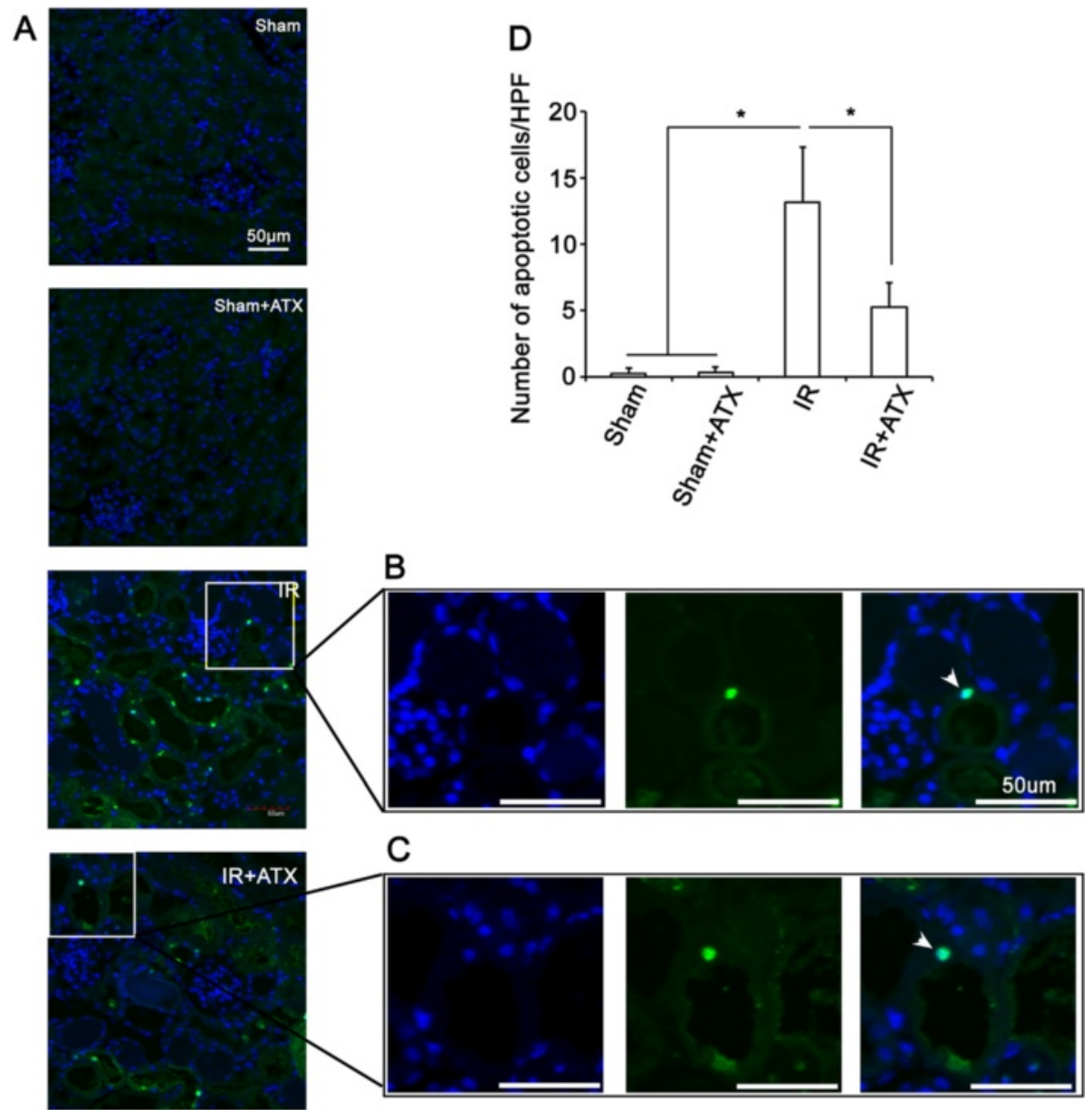

Figure 4 ATX reduces tubular apoptosis after IR. (A) Representative images of TUNEL staining of kidney sections in each experimental groups. (B-C) High magnification of the boxed area. White arrows show TUNEL positive cells. (D) Results of the number of apoptotic cells in kidney sections in each group. *, P $<0.05$ compared with IR group.

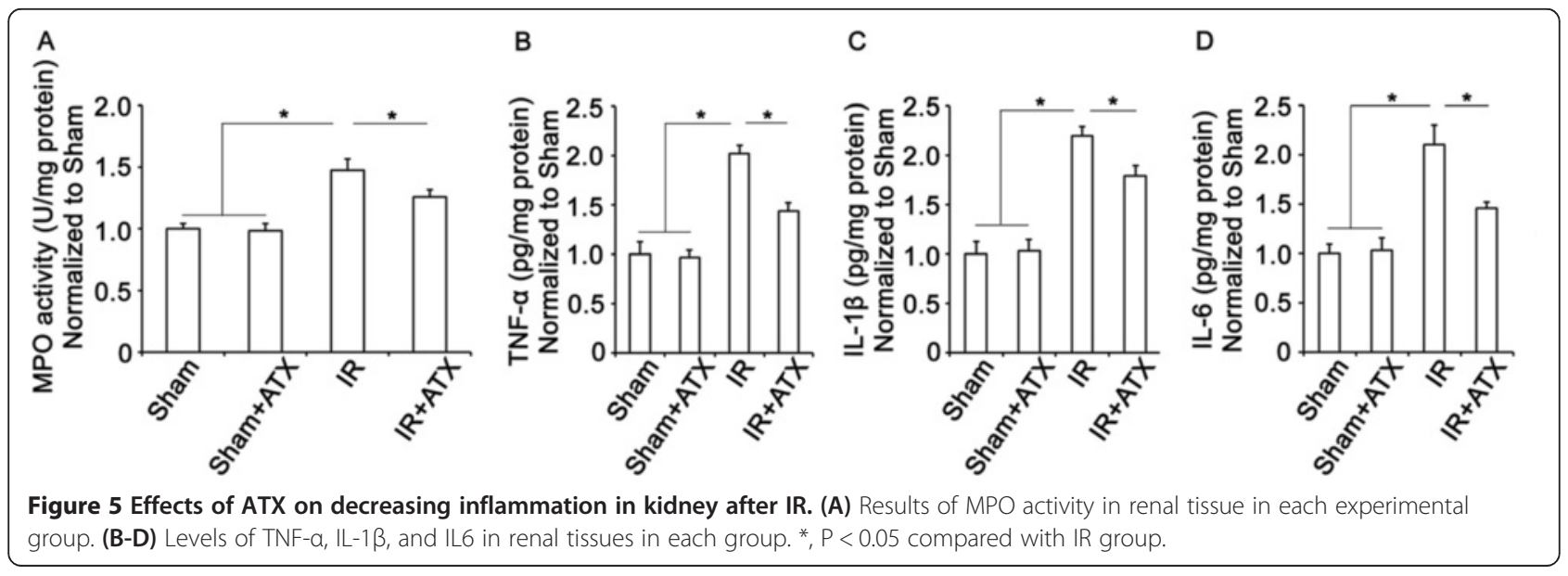



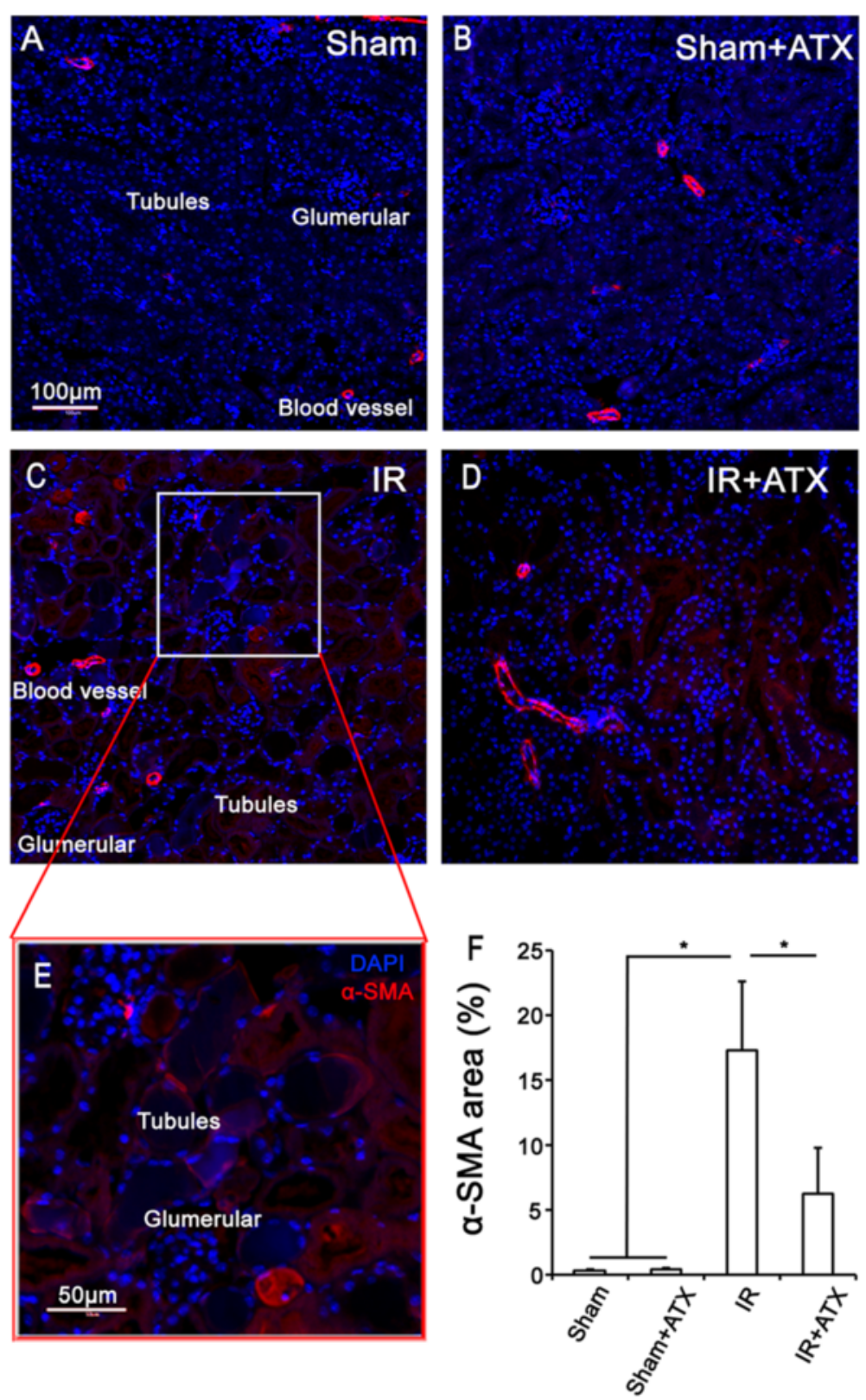

Figure 6 Effects of ATX on reduces the expression of a-SMA in tubular cells after IR. (A-D) Representative images of immunofluorescent staining of a-SMA of kidney sections in each group. (E) High magnification of the boxed area in C further shows the a-SMA expression in renal tissues. (F) Results of the expression of a-SMA in renal sections in each group. * ${ }^{*}<0.05$ compared with IR group.

BUN and $\mathrm{Cr}$ are mainly used to reflect the filtration function of glomerular. Therefore, it gives us inspiration that more sensitive markers might be needed to fully reflect the renal injury after IR.

As well demonstrated, the similar molecular structure to $\beta$-carotenoid gives ATX the capacity to scavenge free radicals and protect oxidative damage to cell membranes, cells, and tissues [15]. ASX can stabilize free radicals by adding them to its long double-bond chain rather than donating an atom or electron to the radical
[25]. In the present study, SOD and MDA, which are commonly used as biomarkers to reflect oxidative stress, were used to evaluate oxidative stress in kidney post IR. Significantly reduced levels of SOD and MDA in the ATX pretreated kidney compared with non-treated controls indicated the powerful free radical scavenging activity of ATX after IR injury (Figure 2), which was consistent with previous studies $[17,18,20]$. Combined with the preserved renal function and renal microstrucutre, it's reasonable to speculate that ATX, a powerful 
antioxidant, might preserve renal function through reducing oxidative stress after IR. More importantly, the specific structure of ATX provides greater antioxidant properties than other antioxidants [26]. ATX contains two additional oxygenated groups on each ring structure, makes ATX highly polar. The polar end groups overlap the polar edges of the all membrane, while the non-polar middle fits the membrane's non-polar interior. This allows ATX to neutralize free radical or other oxidant activity in both the non-polar center of the membrane, as well as along the polar boundary zones of the membrane (Additional file 1: Figure S1) [26,27]. Furthermore, since ATX stabilizes free radicals by adding them to its long double-bond chain rather than donating an atom or electron to the radical, it can resist chain reactions that occur when a fatty acid is oxidized, thus allowing it to scavenge or quench longer than antioxidants that cannot stop the chain reaction [25].

As stated in introduction, oxidative stress, a major contributor to the pathogenesis of IR, leads to tubular apoptosis/necrosis and triggers local inflammatory response in IR injury [7]. In the present study, tubular apoptosis, necrosis (Figure 1 and Figure 3) and inflammation (Figure 4) were shown to be prominent features $24 \mathrm{~h}$ post IR. Pretreatment of ATX significantly reduced tubular apoptosis/necrosis and inflammation after IR injury. Considering the crucial role of oxidative stress in inducing pathological changes of IR and the antioxidant properties of ATX, ATX might alleviate tubular necrosis/ apoptosis and inflammation via scavenging free radicals.

Oxidative stress and inflammation caused by IR could induce EMT $[9,28]$. Although the role of EMT in the pathogenesis of end-stage renal fibrosis is debated, $\alpha$ SMA expression by tubular cells has been suggested to be an early marker of progressive graft damage in kidney transplantation [29]. $\alpha$-SMA is a specific marker for myofibroblasts, the main cellular type involved in tissue fibrosis [30]. In the present study, significantly increased $\alpha$-SMA expression was detected in the tubular cells $24 \mathrm{~h}$ after IR injury, which is consistent with previous study using a swine model [31]. The over-expression of $\alpha$ SMA in tubular cells could partially reflect tubular injury by oxidative stress and inflammation. There is an increasing body of evidence suggesting that IR injury not only causes acute tubular injury but also might be responsible for progressive renal damage leading to worse long-term function [32]. In the present study, pretreatment of ATX, in addition to reducing tubular apoptosis and inflammation, had protective effects in decreasing the expression of $\alpha$-SMA (Figure 5). It might mainly due to the free radical scavenging activity of ATX, and subsequent decreased inflammation. Although the protective effects of ATX on long-term renal fibrosis was not determined in the present study, ATX might have positive effect in protecting kidney from fibrosis via regulating EMT early after IR injury.

\section{Conclusion}

In conclusion, our data showed that pretreatment of ATX was effective in protecting IR-induced acute injury including tubular apoptosis/necrosis, inflammation, and EMT via scavenging free radicals (Additional file 1: Figure S4). Since ATX has been approved by the US FDA in 1999 as a dietary supplement [13], it might be a safe and effective strategy for kidney transplantation in preventing acute injury caused by IR.

\section{Additional file}

Additional file 1: Supplementary materials.

Competing interests

The authors declare that they have no competing interest.

\section{Authors' contributions}

$\mathrm{XQ}$ carried out the molecular studies, and drafted the manuscript. KF, XZ, YY and SZ carried out the animal surgery, histological studies, and statistical analysis. YZ carried out the cell studies and molecular studies. XG and HG revised and approved the manuscript. All authors read and approved the final manuscript.

\section{Acknowledgment}

This study was supported by a grant from the National Natural Science Foundation of China $(81302542,81371207,81171047)$, China Postdoctoral Science Foundation (2014 M551562), and Fundamental Research Funds for the Central Universities (20620140532).

\section{Author details}

'Department of Urology, Affiliated Drum Tower Hospital, School of Medicine, Nanjing University, Nanjing 210008, China. ${ }^{2}$ Institute of Urology, Nanjing University, Nanjing 210093, China. ${ }^{3}$ State Key Laboratory of Pharmaceutical Biotechnology, Nanjing University, Nanjing 210093, China. ${ }^{4}$ Department of Anesthesiology, Affiliated Drum Tower Hospital, School of Medicine, Nanjing University, Nanjing 210008, China.

Received: 5 December 2014 Accepted: 12 January 2015

Published online: 27 January 2015

\section{References}

1. Stallone G, Infante B, Gesualdo L. Older donors and older recipients in kidney transplantation. J Nephrol. 2010;23 Suppl 15:S98-103.

2. Audard V, Matignon M, Dahan K, Lang P, Grimbert P. Renal transplantation from extended criteria cadaveric donors: problems and perspectives overview. Transpl Int. 2008;21:11-7.

3. Pratschke J, Kofla G, Wilhelm MJ, Vergopoulos A, Laskowski I, Shaw GD, et al. Improvements in early behavior of rat kidney allografts after treatment of the brain-dead donor. Ann Surg. 2001;234:732-40.

4. Ojo AO, Wolfe RA, Held PJ, Port FK, Schmouder RL. Delayed graft function: risk factors and implications for renal allograft survival. Transplantation. 1997;63:968-74.

5. Carden DL, Granger DN. Pathophysiology of ischaemia-reperfusion injury. J Pathol. 2000;190:255-66.

6. Devarajan P. Update on mechanisms of ischemic acute kidney injury. J Am Soc Nephrol. 2006;17:1503-20.

7. Perico N, Cattaneo D, Sayegh MH, Remuzzi G. Delayed graft function in kidney transplantation. Lancet. 2004;364:1814-27.

8. Lee DB, Huang E, Ward HJ. Tight junction biology and kidney dysfunction. Am J Physiol Renal Physiol. 2006;290:F20-34.

9. Strutz F, Zeisberg M, Ziyadeh FN, Yang CQ, Kalluri R, Muller GA, et al. Role of basic fibroblast growth factor-2 in epithelial-mesenchymal transformation. Kidney Int. 2002;61:1714-28. 
10. Drugas GT, Paidas CN, Yahanda AM, Ferguson D, Clemens MG. Conjugated desferoxamine attenuates hepatic microvascular injury following ischemia/ reperfusion. Circ Shock. 1991;34:278-83.

11. Atalla SL, Toledo-Pereyra LH, MacKenzie GH, Cederna JP. Influence of oxygenderived free radical scavengers on ischemic livers. Transplantation. 1985;40:584-90.

12. Hussein G, Sankawa U, Goto H, Matsumoto K, Watanabe H. Astaxanthin, a carotenoid with potential in human health and nutrition. J Nat Prod. 2006;69:443-9.

13. Guerin M, Huntley ME, Olaizola M. Haematococcus astaxanthin: applications for human health and nutrition. Trends Biotechnol. 2003;21:210-6.

14. Palozza P, Krinsky NI. Astaxanthin and canthaxanthin are potent antioxidants in a membrane model. Arch Biochem Biophys. 1992;297:291-5.

15. Lim BP, Nagao A, Terao J, Tanaka K, Suzuki T, Takama K. Antioxidant activity of xanthophylls on peroxyl radical-mediated phospholipid peroxidation. Biochim Biophys Acta. 1992;1126:178-84.

16. Shibata A, Kiba Y, Akati N, Fukuzawa K, Terada H. Molecular characteristics of astaxanthin and beta-carotene in the phospholipid monolayer and their distributions in the phospholipid bilayer. Chem Phys Lipids. 2001;113:11-22.

17. Curek GD, Cort A, Yucel G, Demir N, Ozturk S, Elpek GO, et al. Effect of astaxanthin on hepatocellular injury following ischemia/reperfusion. Toxicology. 2010;267:147-53.

18. Lu YP, Liu SY, Sun H, Wu XM, Li JJ, Zhu L. Neuroprotective effect of astaxanthin on $\mathrm{H}(2) \mathrm{O}(2)$-induced neurotoxicity in vitro and on focal cerebral ischemia in vivo. Brain Res. 2010;1360:40-8.

19. Shen H, Kuo CC, Chou J, Delvolve A, Jackson SN, Post J, et al. Astaxanthin reduces ischemic brain injury in adult rats. FASEB J. 2009;23:1958-68.

20. Lauver DA, Lockwood SF, Lucchesi BR. Disodium Disuccinate Astaxanthin (Cardax) attenuates complement activation and reduces myocardial injury following ischemia/reperfusion. J Pharmacol Exp Ther. 2005;314:686-92.

21. Bischoff A, Bucher M, Gekle M, Sauvant C. Differential effect of COX1 and COX2 inhibitors on renal outcomes following ischemic acute kidney injury. Am J Nephrol. 2014;40:1-11.

22. Melnikov WY, Faubel S, Siegmund B, Lucia MS, Ljubanovic D, Edelstein CL. Neutrophil-independent mechanisms of caspase-1- and IL-18-mediated ischemic acute tubular necrosis in mice. J Clin Invest. 2002;110:1083-91.

23. Chen YT, Yang CC, Zhen YY, Wallace CG, Yang JL, Sun CK, et al. Cyclosporineassisted adipose-derived mesenchymal stem cell therapy to mitigate acute kidney ischemia-reperfusion injury. Stem Cell Res Ther. 2013;4:62.

24. Petri $D$, Lundebye AK. Tissue distribution of astaxanthin in rats following exposure to graded levels in the feed. Comp Biochem Physiol C Toxicol Pharmacol. 2007;145:202-9.

25. Kurashige M, Okimasu E, Inoue M, Utsumi K. Inhibition of oxidative injury of biological membranes by astaxanthin. Physiol Chem Phys Med NMR. 1990;22:27-38.

26. Pashkow FJ, Watumull DG, Campbell CL. Astaxanthin: a novel potential treatment for oxidative stress and inflammation in cardiovascular disease. Am J Cardiol. 2008;101:58D-68.

27. Fassett RG, Coombes JS. Astaxanthin: a potential therapeutic agent in cardiovascular disease. Mar Drugs. 2011;9:447-65.

28. Hay ED, Zuk A. Transformations between epithelium and mesenchyme: normal, pathological, and experimentally induced. Am J Kidney Dis. 1995;26:678-90.

29. Badid C, Desmouliere A, Babici D, Hadj-Aissa A, McGregor B, Lefrancois N, et al. Interstitial expression of alpha-SMA: an early marker of chronic renal allograft dysfunction. Nephrol Dial Transplant. 2002;17:1993-8.

30. Liu Y. Epithelial to mesenchymal transition in renal fibrogenesis: pathologic significance, molecular mechanism, and therapeutic intervention. J Am Soc Nephrol. 2004;15:1-12.

31. Simone S, Rascio F, Castellano G, Divella C, Chieti A, Ditonno P, et al Complement-dependent NADPH oxidase enzyme activation in renal ischemia/reperfusion injury. Free Radic Biol Med. 2014;74:263-73.

32. Venkatachalam MA, Griffin KA, Lan R, Geng H, Saikumar P, Bidani AK. Acute kidney injury: a springboard for progression in chronic kidney disease. Am J Physiol Renal Physiol. 2010;298:F1078-94.

\section{Submit your next manuscript to BioMed Central and take full advantage of:}

- Convenient online submission

- Thorough peer review

- No space constraints or color figure charges

- Immediate publication on acceptance

- Inclusion in PubMed, CAS, Scopus and Google Scholar

- Research which is freely available for redistribution

Submit your manuscript at www.biomedcentral.com/submit 